\title{
Découverte d'Ibicella lutea (Lindl.) Van Eselt en Algérie
}

\section{Discovery of Ibicella lutea (Lindl.) Van Eselt in Algeria}

Manuscrit reçu le 6 juin 2020 et accepté le 24 juin 2020

Abdelouahab BelKaSSAM $^{1 *} \&$ Khellaf ReBBAS $^{1,3 \dagger}$, Hicham CHELGHOUM ${ }^{2}$, Mohamed Ait HAMMOU ${ }^{3} \&$ Mohamed Djamel MiARA $^{3}$

\begin{abstract}
${ }^{1}$ Département des sciences de la nature et de la vie, Faculté des sciences, Université Mohamed Boudiaf de M'Sila, Algérie.

${ }^{2}$ Laboratoire de biodiversité végétale : conservation et valorisation, Faculté des Sciences de la nature et de la vie, Université Djillali Liabès de Sidi Bel Abbès, Algérie.

${ }^{3}$ Laboratoire d'Agro-Biotechnologie et de nutrition en zones arides et semi arides. Université Ibn Khaldoun, Tiaret, Algérie.
\end{abstract}

\section{Résumé :}

Les auteurs signalent la découverte à Akbou (Béjaia, NE-Algérie) et à Tissemsilt (W-Algérie) d'ibicelle jaune (Martyniaceae) originaire des zones semi-désertiques d'Amérique du Sud.

Mots clés: Ibicella lutea, découverte, Algérie, Afrique du Nord.

\section{Abstract :}

The authors report the discovery in Akbou (Béjaia, NE-Algeria) and Tissemsilt (W-Algeria) of yellow ibicelle (Martyniaceae) originating in the semi-desert areas of South America.

Key words: Ibicella lutea, discovery, Algeria, North Africa.

\section{Introduction}

Ibicelle jaune est originaire d'Amérique du Sud (Argentine, Brésil, Paraguay, Uruguay). Elle est présente au sud des USA au moins depuis 1914, notamment en Californie (Howell, 1933), Arizona et plus récemment au Mississipi et en Floride où elle peut être envahissante, particulièrement dans les cultures comme le cotonnier, les pâturages, les coupes forestières et en milieu naturel. Elle a été introduite en Australie où elle est considérée comme nuisible («noxious weed», Parsons et Cuthbertson 1992). En Europe, elle a été citée comme occasionnelle en Allemagne (Thellung, 1912), en Belgique (Verloove, 2006), en France (Tison et al., 2014) et en Grèce (DAISIE European Invasive Alien Species Gateway, 2016). Au Royaume-Uni, dans le Yorkshire, Lousley (1958) a identifié des fruits d'Ibicella lutea sur de la laine de mouton importée d'Uruguay en 1956 (Le Bourgeois et al., 2017).

En Afrique du nord, la plante n'est pas citée dans l'index de Dobignard \& Chatelain (2010/2013), alors qu'il cite Proboscidea louisianica, de la même famille. En Algérie, Ibicella lutea n'est pas signalée en Kabylie (Dedeaux, 1894) et elle n'est pas non plus citée

\footnotetext{
${ }^{*}$ Adresse pour le courrier électronique : belkassamabdou@yahoo.fr ; abdelouahab.belkassam@univ-msila.dz

${ }^{\dagger}$ Adresses pour le courrier électronique : rebbas.khellaf@gmail.com $;$ khellaf.rebbas@univ-msila.dz
} 
dans la nouvelle flore d'Algérie (Quezel \& Santa 1962-1963). Les premières mentions de cette espèce datent de 1910,1923, 1924 et 1941, puis elle est à nouveau signalée dans les années 1980 dans la région d'Annaba, et enfin en 2011 dans les prairies de l'aéroport d'Annaba. En 2009, elle est observée en Tunisie dans la vallée de la rivière de Bouhertma (El Mokni et al., 2012).

Plus d'une cinquantaine d'individus d'Ibicella lutea ont été observés dans un champ de céréale, dans la région d'Akbou le 9.8.2018 (Fig. 1) et une deuxième station avec une dizaine d'individus a été observée récemment par (H.C., M.A.H., M.D.M.) dans la région de Tissemsilt (Ouest d'Algérie) le 30.7.2019.

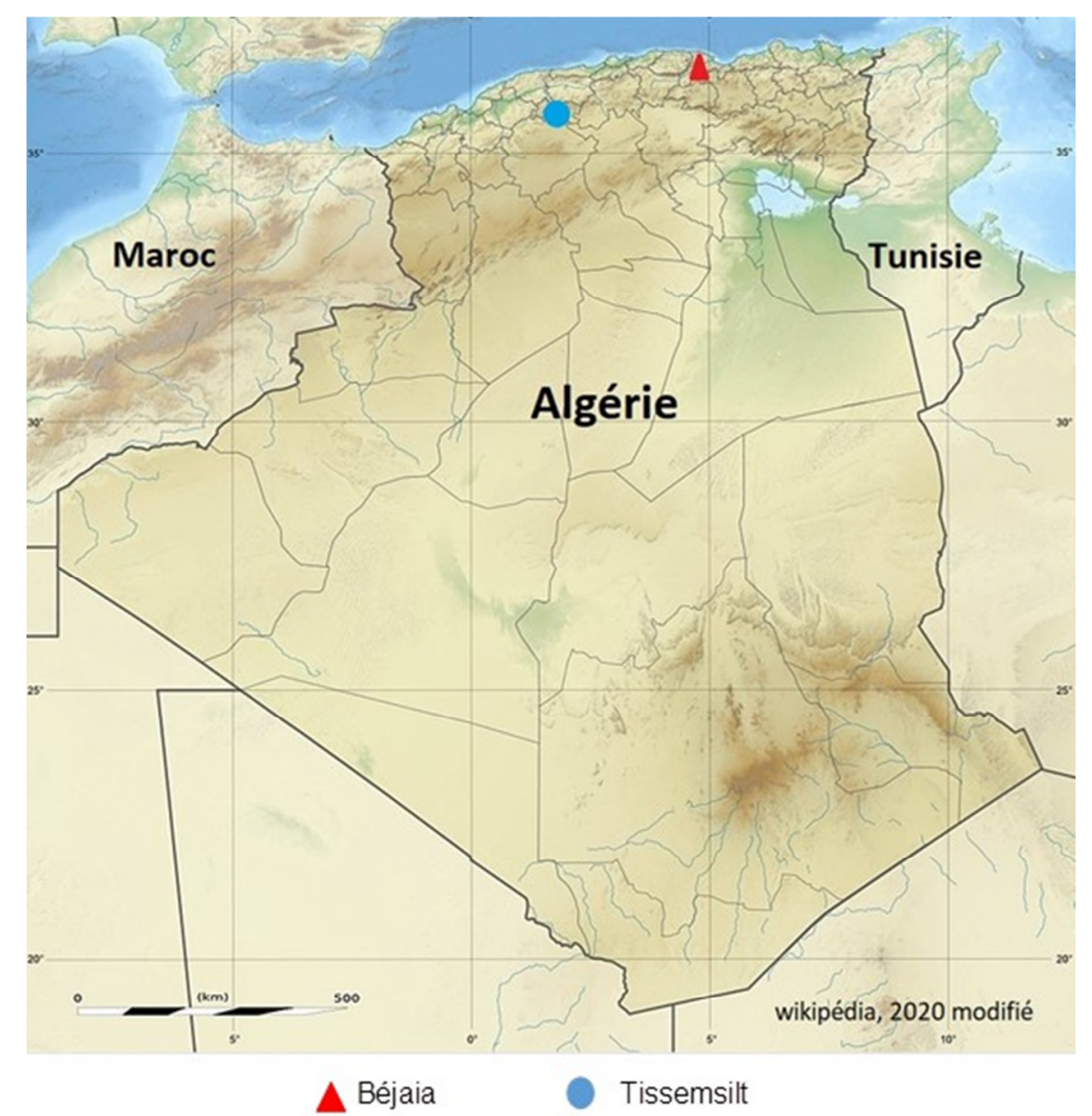

Figure 1 : Localisation géographique des deux stations d'observation d'Ibicella lutea en Algérie

\section{Description botanique}

Ibicella lutea (Lindl.) Van Eselt. (Martynia lutea, Lindl., Martynia montevidensis Cham., Proboscidea lutea (Lind1.) Stapf) est une plante étalée pouvant mesurer 30 à $60 \mathrm{~cm}$ de hauteur et couvrir de 2 à $4 \mathrm{~m}^{2}$ de surface. Elle a des tiges et des feuilles épaisses, plus ou moins collantes, couvertes de poils glanduleux (Fig.2). Les tiges et les feuilles (lorsqu'elles sont écrasées) produisent une substance collante et dégagent une forte odeur très désagréable de viande avariée. Les feuilles sont opposées, longuement pétiolées. Le limbe est suborbiculaire, de grande taille (de 10 à $25 \mathrm{~cm}$ de diamètre), largement cordé à la base à lobes plus ou moins 
imbriqués. La marge est entière à sinueuse, irrégulièrement denticulée. Cinq nervures principales palmées partent de la base. Les deux faces sont couvertes d'une pubescence glanduleuse. L'inflorescence est une grappe dressée comportant 10 à 60 fleurs tubulaires zygomorphes de couleur jaune, tachetées et veinées de rouge. La fleur présente un tube conique de 2 à $3 \mathrm{~cm}$ de long surmonté de 4 lobes dorsaux arrondis et un lobe ventral plus grand. Le fruit est une capsule bivalve ovoïde de 5 à $10 \mathrm{~cm}$ de long et 2,5 à $3,5 \mathrm{~cm}$ de diamètre dans la partie la plus large. Il est prolongé d'un long bec effilé mesurant jusqu'à 14 $\mathrm{cm}$ de long et recourbé en corne de bouquetin. À maturité, le bec se sépare en deux appendices recourbés.

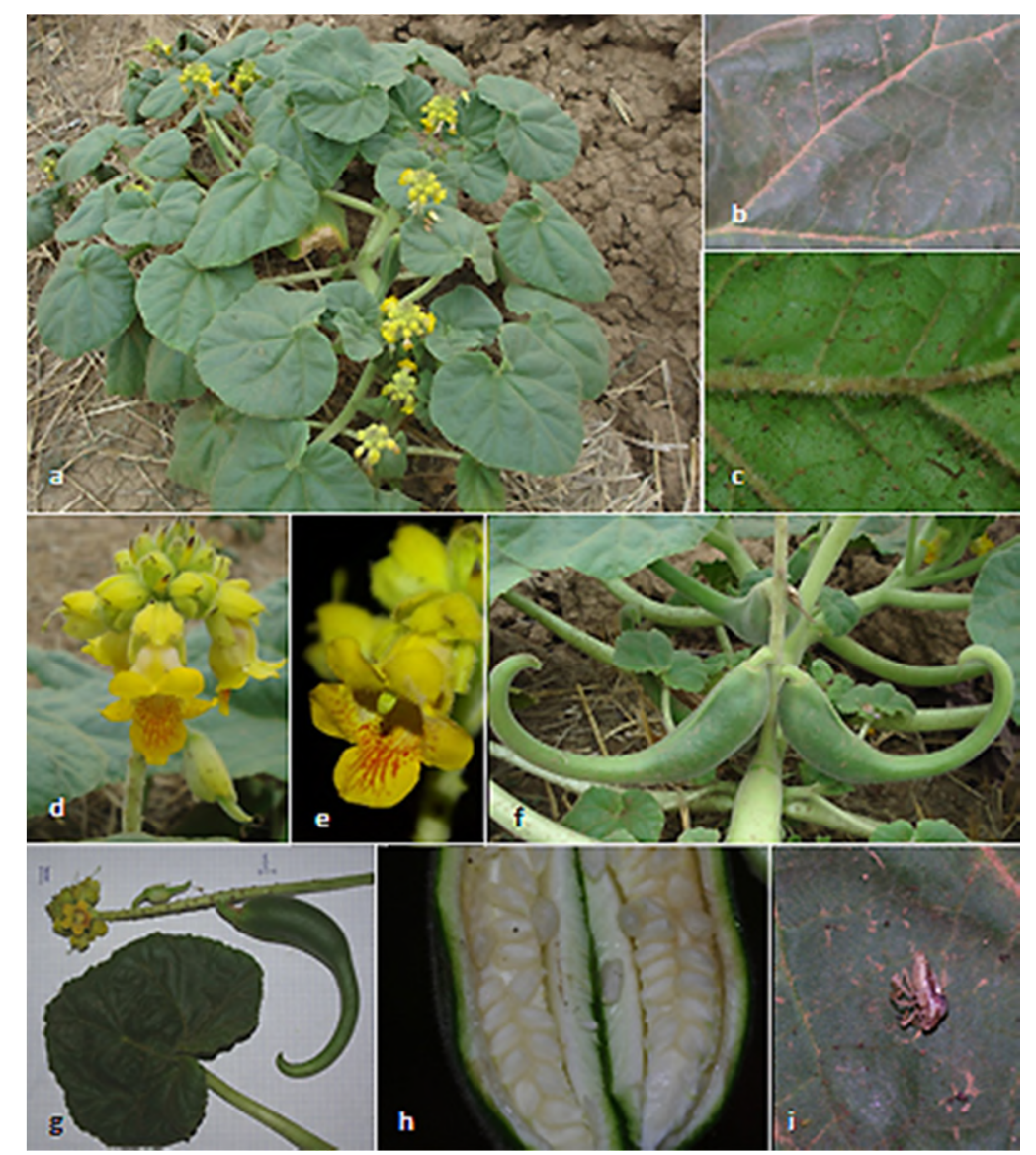

Figure 2 : Illustration d'Ibicella lutea. Akbou (NE-Algérie), 9.8.2018 (photos K. Rebbas)

Légende : (a) Aspect et port de la plante dans son milieu (sol sec et pauvre), (b) et (c) Aspect poilu-glanduleux des feuilles (face supérieure et face inférieure), (d) Inflorescences multiflores en épi, (e) Fleur à corolle jaune d'or en forme de trompette couverte de cils glanduleux, (f) Détail sur la forme du fruit durant la période de maturation, (g) Détail d'une feuille (la largeur peut varier de 10 à $25 \mathrm{~cm}$ ), de la fleur et du fruit sur papier millimétré, (h) Graines avant maturation. (i) Insecte piégé sur la feuille.

La capsule est d'abord charnue, puis le péricarpe se dessèche et se détache laissant une capsule lignifiée au tégument très dur, couverte d'épines rigides de 1 à $6 \mathrm{~mm}$ de long. La capsule s'ouvre par un orifice situé à la base des deux appendices, libérant quelques dizaines (50 à 100) de graines noires ovales aplaties, longues de $10 \mathrm{~mm}$ et larges de 5 à $6 \mathrm{~mm}$, à tégument tuberculé. La plantule présente des cotylédons courtement pétiolés au limbe 
rectangulaire formant deux bandes longitudinales séparées par une nervure médiane déprimée. Les premières feuilles sont opposées, courtement pétiolées, à limbe suborbiculaire, cordé à la base et plus ou moins charnu. Limbe et pétiole sont couverts de poils glanduleux (Le Bourgeois et al., 2017).

\section{Biologie et reproduction}

Ibicella lutea est une espèce annuelle ; elle se multiplie par graines. Elle germe au printemps, puis fleurit et fructifie durant l'été. Un plant peut produire jusqu'à 122 fruits, contenant chacun 71 graines (Riffle et al., 1989). En Tunisie et en France, il semble que seulement un tiers des fleurs soient fertilisées et produisent un fruit. De même, sur les individus observés en Algérie, seules les fleurs situées dans le tiers inférieur de la grappe ont donné des fruits, les autres fleurs ont avorté.

\section{Habitat et écologie}

Cette espèce se développe sur tout type de sol qu'il soit riche ou pauvre, mais on la retrouve fréquemment sur les sols pauvres, acides et temporairement asséchés dans les régions à climat semi-aride. Elle pourvoit à ses besoins en eau grâce à l'humidité contenue dans l'air. En Tunisie, elle se développe dans les vallées humides, mais desséchées en été, au nord-ouest du pays, sur les sols alluviaux relativement pauvres en matière organique (Shoar-Ghafari \& Vintéjoux, 2000; El Mokni et al., 2012).

Le mode de piégeage d'Ibicella lutea est dit "passif", car il ne nécessite pas de mouvement pour attraper sa proie. Les poils glanduleux qui la recouvrent sécrètent un mucilage adhésif permettant d'engluer les insectes ailés (essentiellement de petits diptères) qui se posent sur elle. Une seule feuille peut capturer à elle seule plus d'une centaine d'insectes.

Elle est considérée comme une "plante protocarnivore". Des études ont démontré que l'activité de protéases à l'intérieur du mucilage était inexistante, ce qui tend à valider la précédente affirmation (Rice, 1999).

\section{Intérêt économique}

Cette espèce a des propriétés très intéressantes en matière de lutte biologique contre les insectes ravageurs de cultures, tels que les pucerons et aleurodes. Les individus ailés sont capturés par la plante qui limite ainsi l'expansion de leur population sur la culture.

Elle génère également beaucoup d'attention en recherche pharmacologique pour ses propriétés anti-bactériennes. Ibicella lutea pousse à l'état sauvage en Uruguay où elle est utilisée en médecine populaire comme antiseptique pour les infections des yeux et de la peau.

Dans un dépistage antérieur, il présentait un large spectre antibactérien. À partir de l'extrait chloroformique de la plante, le composé antibactérien principal : 11-O-(6'-O-acetyl- $\beta$-dglucopyranosyl)-stearic acid a été isolé et identifié par plusieurs méthodes MS et RMN en tant que nouveau composé (Cerdeiras et al., 2000). 
L'extrait de I. lutea a eu un effet sur le taux de croissance bactérien et la morphologie bactérienne. Elle a également affecté la différenciation de l'essaimage, l'hémagglutination et la formation de biofilm de Proteus mirabilis sur le verre et le polystyrène. Ces résultats suggèrent qu'I. Lutea pourrait jouer un rôle d'agent de lutte contre les infections urinaires à $P$. mirabilis (Sosa \& Zunino, 2009).

Les esters gras sont l'une des alternatives les plus viables, car ils ont des propriétés similaires à celles des combustibles fossiles et ils peuvent être utilisés comme substituts des combustibles conventionnels sans apporter de grandes modifications aux moteurs. Les huiles obtenues des graines d'Ibicella lutea ont des propriétés intéressantes en tant que sources de biocarburants (Bolonio et al., 2017).

Dans le domaine alimentaire, les fleurs voyantes et parfumées et les fruits aux formes étranges de Proboscidea, Ibicella et Martynia ont mené à leur culture comme plantes ornementales aux États-Unis et en Europe. Les noms communs des plantes martyniacées dans les langues européennes sont généralement des variantes de la griffe du diable, de la griffe du chat ou des plantes à licorne. Aux États-Unis, en Amérique du Sud et en Europe, les jeunes fruits de Proboscidea et d'Ibicella sont consommés sous forme de légumes et de cornichons (Bretting, 1984).

Dans certaines régions, comme en Australie-Occidentale, la plante est considérée comme une mauvaise herbe nuisible car les griffes peuvent blesser le bétail.

\section{Conclusion}

Cette découverte enrichit d'avantage la flore vasculaire Algérienne d'origine exotique. Cette espèce doit être surveillée car elle pourrait rapidement devenir envahissante en Algérie.

\section{Remerciements}

Le deuxième auteur $(\mathrm{KR})$ tiens à remercier vivement $M$ Errol Véla (Univ. de Montpellier) pour ses conseils.

\section{Références}

Bolonio D., Houachri T., Llamas A., Al-Lal AM., Rodríguez-Fernández J., El Gazza M. et al. 2017. Fatty acid methyl esters (FAMEs) obtained from rare seeds of Tunisia: Ibicella lutea, Peganum harmala, Smyrnium olusatrum, Onopordum nervosum and Solanum elaeagnifolium. J Fundam Renewable Energy Appl, 7:4 (Suppl)

Bretting PK., 1984. Folk names and uses for martyniaceous plants. Economic Botany, 38: 452-463.

Cerdeiras MP., Fernández J., Soubes M., Vero S., Ferreira F., Moyna P. et al., 2000. A new antibacterial compound from Ibicella lutea. Journal of Ethnopharmacology, 73 (3) : 521-525. DAISIE European Invasive Alien Species Gateway, 2016. Ibicella lutea. Available from: http://www.europe-aliens.org/speciesFactsheet.do? speciesId=19514\# 
Debeaux OM., 1894. Flore de la Kabylie du Djurdjura, ou Catalogue méthodique et raisonné de toutes les plantes vasculaires et spontanées observées jusqu'à ce jour dans cette contrée Paris, Libr. Sci. Nat. : 484p.

Dobignard A. \& Chatelain C., 2010-2013. Index synonymique de la flore d'Afrique du Nord. Genève.

El Mokni R., Hamdi N., De Belair G. \& Hédi El Aouni M., 2012. Découverte d'Ibicella lutea (Lindl.) Van Eselt. (Martyniaceae) en Kroumirie (Nord-Ouest de la Tunisie). Poiretia, 4 : 1-6. Howell JT., 1933. Plants worthy of note - III. Leaflets of Western Botany, 1 (5) : 39-40.

Le Bourgeois T., Rodriguez A., Vela E., Marnotte P. \& Fried G., 2017. Focus sur une espèce: Ibicella lutea (Lind1.) Van Eselt. Nouvelle observation d'une curieuse plante dans la Haute Garonne. Journal de Botanique, 79 : 53-55.

Lousley JE., 1958. Alien plants introduced by the Yorkshire Wool Industry. The Naturalist, $866: 77-91$.

Parsons WT. \& Cuthbertson EG., 1992. Noxious weeds of Australia. Inkata Press, Melbourne (AU), 692 p.

Quézel P. \& Santa S., 1962-1963. Nouvelle flore d'Algérie et des régions désertiques méridionales. Ed. CNRS, Paris (FR),Tome I:1-565, Tome II: 566-1170.

Rice B., 1999. Testing The Appetites of Ibicella and Drosophyllum. Carnivorous Plant Newsletter (CPN), 28 (2): 40-43.

Riffle MS., Thilsted WE., Murray DS., Ahring RM. and Waller GR., 1988. Germination and seed production of unicorn-plant (Proboscidea louisianica). Weed Science, 36 : 787-791.

Thellung A., 1912. La flore adventice de Montpellier. Mémoires de la Société des Sciences Naturelles de Cherbourg 38.

Shoar-Ghafari A. \& Vintéjoux C., 2000. Morphologie des organes de capture des plantes carnivores. Acta Botanica Gallica: Botany Letters, 147: 37-59.

Sosa V. \& Zunino P., 2009. Effect of Ibicella lutea on uropathogenic Proteus mirabilis growth, virulence, and biofilm formation. Journal of Infection in Developing Countries, 3(10):762-770.

Tison J-M., Jauzein P. \& Michaud H. 2014. Flore de France méditerranéenne continentale.

Naturalia Publications, Turriers, Conservatoire Botanique National Méditerranéen de Porquerolles, Hyères, France.

Verloove F., 2006. Catalogue of neophytes in Belgium (1800-2005). Scripta Botanica

Belgica, $39: 89 \mathrm{p}$. 